Zentralatom kann außer Zahl und Abstand keine bestimmte Aussage gemacht werden. Da in der Flüssigkeit schon die zweite Koordination mit 4 Atomen nicht die gleiche Besetzung wie im Kristall hat, kann nicht auf die Existenz von kleinsten Kriställchen in derselben geschlossen werden, denn solche sind nach früherer Definition durch die periodische Aneinanderreihung einer kleinsten festen Struktureinheit in den 3 Richtungen des Raumes charakterisiert. Es ist vielmehr' zu vermuten, daß die Nachbaratome in der Flüssigkeit trotz der teilweise vorhandenen Bindungskräfte noch eine Bewegungsfreiheit in bezug auf das Zentralatom haben und daß auch Platzwechsel möglich sind.

Zum Vergleich mit der neu ermittelten Verteilungsfunktion $W$ sind in Abb. 3 die Wahrscheinlichkeitskurven nach Menke und nach Boyd und Wakeham wiedergegeben. Erstere zeigt eine völlig gleichmäßige Verteilung mit 3 Maxima, von denen das erste bei 3,26 A liegt, und das nach eigener Auswertung eine Koordinationszahl von 12 Atomen liefert. Es ist leicht möglich, daß bei der Festlegung der $W$-Kurve die Existenz eines
Zwischenmaximums unbemerkt bleibt, wenn nicht genügend Integralwerte graphisch oder mit Hilfe des harmonischen Analysators bestimmt werden. Tatsächlich zeigte eine an Hand der M e n k e schen Intensitätskurve ${ }^{6}$ selbst ermittelte Verteilungsfunktion $W$ in der Umgebung von 4 À einige Zwischenmaxima.

Boyd und Wakeham ${ }^{9}$ dagegen ermittelten aus ihrer Verteilungsfunktion $\left(+30^{\circ} \mathrm{C}\right)$ einen kleinsten Atomabstand von 2,88 ^ und eine Koordinationszahl von 6 Atomen. Es ist sehr unwahrscheinlich, daß bei gleicher Koordinationszahl der kleinste Atomabstand in der Flüssigkeit kleiner sein soll als im Kristall, und schon dieses Ergebnis weist darauf hin, daß die experimentell gewonnenen Unterlagen nicht richtig sein können.

Gerade weil Quecksilber schon bei Raumtemperatur nach den obigen Ergebnissen, wenigstens in kleinsten Bereichen, eine dem Kristall verwandte Atomverteilung aufweist, wäre es recht aufschlußreich, die Veränderungen des Interferenzbildes und der Atomverteilung mit der Temperatur unter einwandfreien Versuchsbedingungen zu untersuchen.

\title{
Bemerkung zur klassischen Magnetostatik
}

\author{
Von HeInRICh Ottr \\ (Z. Naturforschg. 3 a, 422-425 [1948]; eingegangen am 11. Mai 1948)
}

\begin{abstract}
Es wird folgende Schwierigkeit behoben: Das Feld $\mathfrak{H}$ eines Elementarmagneten weicht im homogenen Medium von der Permeabilität $\mu$ erfahrungsgemäß um den Faktor $\mu_{0} / \mu$ von seinem Vakuumwert ab. In diesem Punkt scheint das klassische Modell des Ampèreschen Molekularstroms zu versagen, da in der 1. Hauptgleichung $\mu$ gar nicht vorkommt.
\end{abstract}

$\mathrm{B}$ ringt man einen magnetischen Dipol, dargestellt durch einen Ampèreschen Molekularstrom, in ein homogenes, unendlich ausgedehntes Medium von der Permeabilität $\mu$, so bleibt sein Feld $\mathfrak{S}$, wie man aus der ersten Hauptgleichung rot $\mathfrak{S}=j$ schließen möchte, völlig ungeändert, während wir doch andererseits für einen eingebetteten Dipol erfahrungsgemäß eine Feldänderung um den Faktor $\mu_{0} / \mu$ fordern müssen. Somit scheint also das klassische Modell des Molekularstroms keine adäquate Darstellung eines Elementarmagneten zu sein, eine Schwierigkeit, die zwar nicht unbekannt ist, deren befriedigende Lösung

1 Würzburg, Franz-Schubert-Str. 3. ich aber in den Lehrbüchern, soweit darauf überhaupt eingegangen wird, nirgends finden konnte.

Wir stellen im folgenden die beiden Dipolpotentiale im Medium, wie sie sich aus den zwei verschiedenen Interpretationen des statischen Magnetismus ergeben, einander gegenüber:

1. Das Potential eines Molekularstroms $J$ von der Windungsfläche $f$

$$
4 \pi \psi_{1}=J \frac{(f \mathfrak{r})}{r^{3}} .
$$

das man bekanntlich aus der 1. Hauptgleichung mittels der Äquivalenz von Stromschleife und 
Doppelfläche gewinnt und das, wie bereits betont, explizit unabhängig von $\mu$ ist;

2. das Potential einer magnetischen Doppelquelle vom Moment $\mathfrak{p}$

$$
4 \pi \psi_{2}=\frac{1}{\mu} \frac{(p r)}{r^{3}}
$$

das man in der Regel auf dem Boden der magnetischen Fluidumshypothese ableitet bzw. der Elektrostatik entlehnt und das vermöge des Nenners $\mu$ die Feldänderung richtig zum Ausdruck bringt, weil das Moment $\mathfrak{p}$ gemäß seiner Definition als Ladung $\times$ Abstand (bzw. als Grenzwert dieses Produkts) unabhängig von $\mu$ ist, seinen Vakuumwert also in jedem beliebigen Medium beibehält (,Erhaltung der magnetischen Ladung“). Wir werden im folgenden $\mathfrak{p}$ das ,,magnetostatische Moment" des Dipols nennen, hingegen das in Gl. (1) auftretende Produkt $J f$ (Strom $\times$ Fläche) als das „elektrodynamische Moment“ des Dipols bezeichnen.

Soll $\psi_{1}$ mit der Erfahrung und mit $\psi_{2}$ in Einklang sein, so muß zwischen den beiden Momenten in jedem beliebigen homogenen Medium die Beziehung

$$
J f_{\mu}=\mathfrak{p}=\mathrm{const}
$$

bestehen. Was soll man aber von dieser Beziehung halten, kann man sie irgendwie begründen? Nun keinesfalls so, wie da und dort zu lesen steht, daß nämlich infolge Abschirmung durch irgendeinen ,freien“ Magnetismus das in Gl. (1) vorkommende, aus dem Äquivalenzsatz resultierende Moment $\boldsymbol{J} f$ der Windungsfläche des Stromes im Medium anders anzusetzen sei als im Vakuum. Abgesehen davon, daß eine solche nachträgliche Änderung im Äquivalenzsatz mathematisch unzulässig ist, trifft der Hinweis auf den Äquivalenzsatz auch nicht den Kern der Sache: denn die in Rede stehende Schwierigkeit stellt sich ja nicht erst im Zusammenhang mit der Äquivalenz von Strom und Doppelfläche ein, ihre Wurzel liegt bereits in der bündigen Aussage der 1. Hauptgleichung, nach welcher das Feld $\mathfrak{S}$ eines Stromes unabhängig von der Permeabilität des Einbettungsmaterials ist, ganz gleich, wie man es berechnet, sei es aus einem Vektorpotential oder über den Äquivalenzsatz oder irgendwie anders

Demnach hat es also zunächst den Anschein. als sei das Modell des Molekularstroms überhaupt kein vollwertiger Ersatz für das Modell der
Doppelquelle, da wir beim Wechsel des Einbettungsmaterials jeweils ein neues Modell bereithalten bzw. das Moment $J f$ des Molekularstroms jeweils eigens auf den $\mu_{0} / \mu$-ten Teil seines Vakuumwertes reduzieren müßten. Wir werden im folgenden aber zeigen, daß es die Natur selbst ist, welche uns diese Reduktion abnimmt, nämlich durch eine Rückwirkung des Einbettungsmaterials auf den Strom. Daß eine solche Rückwirkung vorhanden ist, macht man sich leicht klar, denn das Heranführen des Einbettungsmaterials an den Molekularstrom ändert den Fluß von $\mathfrak{B}$ und erzeugt Induktionsspannungen, die am Strom angreifen und ein (,diamagnetisches") Moment induzieren. Wie die strenge Rechnung zeigt, ändert sich dabei der Strom gerade reziprok zu $\mu$, so daß das Produkt $J f \mu$ im jeweiligen Einbettungsmaterial ein und dieselbe Konstante darstellt, die nun als das gleichbleibende statische Moment $\mathfrak{p}$ einer Doppelquelle aufgefaßt werden kann. Dies ist der tiefere Sinn der Beziehung (3). Daß diese Beziehung eine Anderung des Molekularstroms involviert und daß diese Änderung auf der Rückwirkung des Einbettungsmaterials beruht, findet sich selbst in sonst vortrefflichen Darstellungen nirgends ausgesprochen, geschweige denn bewiesen.

Es wäre umständlich, wollte man den Beweis von Gl. (3) an die oben erwähnten Induktionsspannungen anknüpfen; wir kommen dem Problem leichter von der energetischen Seite bei. Da$\mathrm{zu}$ erinnern wir an die bekannte Energiebilanz des magnetischen Feldes konstanter Ströme: Führt man in ein Solenoid eine paramagnetische Substanz ein, so gewinnt man wegen der gegenseitigen Anziehung mechanische Arbeit, die aber bei konstant gehaltenem Strom nicht aus der Feldenergie ( $\eta={ }_{2}^{\mu} H^{2}$ wächst ja sogar an), sọndern durch die treibende EMK des Stromes gedeckt wird, welche zum Ohmschen Widerstand nun noch die Induktionsspannungen zu überwinden hat. Da beim Molekularstrom aber keine treibende EMK vorhanden ist, geht in diesem Fall die Arbeit auf Kosten der Feldenergie, was dadurch bewerkstelligt wird, daß der Strom bei Annäherung des Mediums infolge Induktion sinkt und in der Endlage des Einbettungsmaterials auf dem erreichten niedrigeren Wert stehenbleibt.

Zur Durchführung der Rechnung benutzen wir ein einfaches Modell: Ein unendlich langes 
Solenoid vom Radius $R$ werde vom Strom $J / \mathrm{cm}=H$ durchflossen. Eine magnetische Flüssigkeit, welche von außen durch den Spulenmantel einströmen kann, sei in das Spuleninnere vorgedrungen, lasse aber noch einen koaxialen $\mathrm{Zylinder} Z \mathrm{Z}$ vom Radius $r<R$ frei. Die Feldenergie pro $\mathrm{cm}$ Spulenlänge ist dann

$$
\begin{aligned}
E=\frac{H^{2}}{2}\left\{, \mu\left(\pi R^{2}-\pi r^{2}\right)+\mu_{0} \pi r^{2}\right\} & \\
= & \frac{J^{2} \pi}{2}\left\{\mu R^{2}+\left(\mu_{0}-\mu\right) r^{2}\right\} .
\end{aligned}
$$

Beiderseits der Mantelfläche von Z, die wir uns etwa als arbeitleistende elastische Membran vorstellen wollen, greifen die Maxwellschen Drucke $p=\eta$ an, welche bei einer Vergrößerung von $r$ um $d r$ die Arbeit

$$
\begin{aligned}
d A=\left\{\eta\left(\mu_{0}\right)-\eta(\mu)\right\} 2 \pi r d r & \\
& =\frac{H^{2}}{2}\left(\mu_{0}-\mu\right) 2 \pi r d r .
\end{aligned}
$$

leisten. Wir nehmen nun an, daß der Strom $g$ nicht durch eine EMK unterhalten werde, sondern widerstandslos verlaufe, und beziehen ihn in die Energiebilanz mit ein. Da wir für langsame Änderungen nach wie vor $H$ durch $J$ ersetzen dürfen, lautet die Änderung der Feldenergie (4):

$$
\begin{array}{r}
d E=J d J \pi\left\{u R^{2}+\left(\mu_{0}-\mu\right) r^{2}\right\} \\
+J^{2} \pi\left(\mu_{0}-\mu\right) r d r .
\end{array}
$$

Der Energiesatz $d A+d E=0$ führt dann mit Gl. (5) und Gl. (6) zur Differentialgleichung für $J$ :

$$
\begin{aligned}
J d J\left\{u R^{2}+\left(\mu_{0}-\mu\right) r^{2}\right\} \\
+2 J^{2}\left(\mu_{0}-\mu\right) r d r=0
\end{aligned}
$$

oder $\frac{d J}{J}=-\frac{\left(\mu_{0}-\mu\right) d r^{2}}{\mu R^{2}+\left(\mu_{0}-\mu\right) r^{2}}$ mit der Lösung

$$
J=\frac{C}{\mu R^{2}+\left(\mu_{0}-\mu\right) r^{2}} .
$$

Die Integrationskonstante $C$ bestimmt sich aus den Anfangswerten. Für $r=R$ ist das Spuleninnere frei vom Medium, der zugehörige Strom sei $J_{0}$, also $C=J_{0} \mu_{0} R^{2}$. Für die vom Medium völlig ausgefüllte Spule $(r=0)$ lautet dann der Strom

$$
J={ }^{\prime \prime}{ }^{\prime \prime} J_{0} .
$$

Damit ist die Beziehung (3) bewiesen. Überraschenderweise gilt sie auch noch für jeden Zwischenwert von $r$ zwischen 0 und $R$, m. a. W. für ein geschichtetes Medium; denn unter Einführung einer mittleren Permeabilität $\bar{\mu}$, die sich additiv aus den jeweiligen Volumenanteilen des Vakuums und des Mediums zusammensetzt.

$\bar{"}=\frac{\mu_{0} \pi r^{2}+\mu\left(\pi R^{2}-\pi r^{2}\right)}{\pi R^{2}}=\frac{\mu R^{2}+\left(\mu_{0}-\mu\right) r^{2}}{R^{2}}$,

folgt aus Gl. (7) nach Division durch $J R^{2}$ sofort: $\bar{\mu} d J+J d \bar{\mu}=d(\bar{\mu} J)=0$. Diese einfache Rückwirkung des geschichteten Mediums auf den Strom ist jedoch eine Ausnahme, die auf dem besonderen Kraftlinienverlauf unseres Beispiels beruht; die Bedingung, unter welcher Gl. (3) allgemein für geschichtete oder inhomogene Medien zutrifft, werden wir im letzten Absatz kennenlernen.

Es bedarf wohl kaum eines besonderen Hinweises, daß die Invarianz von $J f \mu$ gegenüber dem Wechsel des Einbettungsmaterials grundsätzliche Bedeutung hat, wird doch dadurch eine ,Erhaltung der magnetischen Ladung" vorgetäuscht, ansonsten die Fluidumshypothese beim Wechsel des Einbettungsmaterials sofort Schiffbruch erleiden müßte. Und umgekehrt läßt sich die unerquickliche Fluidumshypothese nur unter dem Gesichtspunkt dieser Invarianz völlig ausschalten, da nun die Doppelquelle auch im Medium widerspruchslos durch das Modell des Molekularstroms ersetzt werden kann.

Um einem Irrtum vorzubeugen, mag noch eine Bemerkung am Platze sein. Die Schwächung des elektrooder magnetostatischen Feldes im Medium (z. B. beim Coulombschen Gesetz) läßt sich bekanntlich auf die Abschirmung durch die ,freien“ Ladungen am Rande des Einbettungsmaterials zurückführen, aber es wäre ganz abwegig, wollte man nach einem ähnlichen Abschirmungseffekt für das Feld des Molekularstroms suchen. Man könnte dabei irrtümlich auf den Lorentzschen Magnetisierungsstrom rot $\mathfrak{M}$ verfallen, der z. B. am Rand unseres Spulenkerns auftritt. Dieser setzt sich mit dem Spulenstrom i zum Gesamtstrom $\mathfrak{g} \mathfrak{v}=\mathfrak{j}+$ rot $\mathfrak{M}$ der Elektronentheorie zusammen, der nun seinerseits die Mikrofeldstärke $\mathfrak{h}$ bestimmt (rot $\mathfrak{h}$ $=\mathfrak{Q} \mathfrak{b})$. Im paramagnetischen Medium ist der Magnetisierungsstrom aber gleichsinnig mit $J$, schwächt also nicht den Spulenstrom, sondern verstärkt ihn (auf den Betrag $J \mu / \mu_{0}$, wie man leicht nachrechnet). Infolgedessen wird auch $\mathfrak{h}$ verstärkt; für die Maxwellsche Makrofeldstärke $\mathfrak{S}$ besagt dies aber unmittelbar noch nichts, denn die gemittelte Mikrofeldstärke $\mathfrak{h}$ ist nicht $\mathfrak{C}$, sondern $\mathfrak{B} / \mu_{0}$, und unsere Überlegung führt somit zum trivialen Ergebnis, daß im Medium eben die Induktion $\mathfrak{B}$ um den Faktor $\mu / \mu_{0}$ vergrößert ist. Die- 
Schwächung des Molekularstromfeldes im Medium ist also nicht durch einen Abschirmungseffekt der Umgebung zu erklären, sondern, im Gegensatz zur Elektrostatik und der Fluidumsmagnetostatik, nur durch einen induktiven Eingriff in den Strom selbst.

Kürzer, aber weniger instruktiv als mit unserem Spulenmodell läßt sich Gl. (3) mittels der allgemeinen Energieprinzipien der Elektrodynamik beweisen, und zwar für einen Molekularstrom von beliebiger Form. Aus der Stromenergie eines eingebetteten Stromes

$$
T=\frac{\mu}{2} L^{0} J^{2}
$$

worin $L^{0}$ das Selbstpotential im Vakuum bedeutet, folgt, wenn sich $\mu$ in jedem Punkt des Raumes um $d \mu$ ändert:

$$
d T=\| L^{0} J d \cdot J+\frac{1}{2} L^{n} J^{2}{ }^{\prime} \mu .
$$

Die dabei geleistete mechanische Arbeit schreibt sich, wenn wir $\mu$ als generalisierte Koordinate einführen, $d A=Q_{\mu} d \mu$ mit der generalisierten $\mathrm{Kraft}^{2} Q_{\mu}=\left(\frac{\partial T}{\partial \mu}\right)_{J}$. Also ist

$$
d A=\frac{1}{2} L^{0} J^{2} d \mu
$$

und der Energiesatz $d A+d T=0$ führt nun sofort $\mathrm{zu}$

$$
\mu d J+J d \mu=d\left(J_{\mu}\right)=0 .
$$

Unser Beweis bezieht sich nur auf ein homogenes, unendlich ausgedehntes Medium; im inhomogenen oder geschichteten Medium ist der Molekularstrom nicht unter allen Umständen einer Doppelquelle äquivalent, wie das folgende Beispiel zeigen möge. Wir wollen einen magneti-

2 Vgl. etwa Cl. S cha ef er, Einführung in die Theoret. Physik, 1II/1, Leipzig 1932, S. 276. schen Dipol mit einem homogenen Medium umgeben, das den ganzen Raum außerhalb einer konzentrischen Kugel vom Radius $R$ erfülle. Das Feld innerhalb dieser Kugel, das sich aus dem primären Dipolfeld und dem durch die Magnetisierung des Mediums erzeugten homogenen Feld zusammensetzt, fällt nun verschieden aus, je nachdem man sich den Dipol als Doppelquelle oder als Molekularstrom denkt. Denn im ersten Fall ist das primäre Dipolfeld gleich dem unveränderten Vakuumfeld der Doppelquelle zu setzen, im zweiten Fall hat man aber die Rückwirkung des Mediums auf den Molekularstrom zu berücksichtigen. Diese Rückwirkung findet man, wie oben, mit Hilfe des Energiesatzes, wobei man im Interesse der Rechnung den Molekularstrom zweckmäßig als eine rotierende Kugelschale vom Radius $R_{0}$ mit gleichförmiger elektrischer Flächenladung idealisiert. Wir wollen hier nur das Ergebnis der Rechnung anführen: Solange das Medium noch weit vom Molekularstrom entfernt ist $\left(R_{0} \ll R\right)$, ist die Rückwirkung proportional zur 3. Potenz von $R_{0} / R$ und daher in allen makroskopischen Experimenten unmeßbar klein; zieht man die Hohlkugel $R$ aber auf den Molekularstrom zusammen, so wird die Rückwirkung merklich, sobald das Einbettungsmaterial nahe an den Strom herankommt ( $R \approx R_{0}$ ) oder gar in die Windungsfläche eindringt $\left(R<R_{0}\right)$. Schließlich erreicht das dynamische Moment. $J f$ des Stromes den vorhin gefundenen Grenzwert $J_{0} f \mu_{0} / \mu$, wenn das Einbettungsmaterial den ganzen Innenraum des Molekularstroms ausfüllt $(R=0)$. Wir entnehmen diesem Beispiel zugleich, daß das Modell des Molekularstroms im geschichteten oder inhomogenen Medium nur dann völlig äquivalent dem einer Doppelquelle ist, wenn man die Windungsfläche (in unserem Beispiel den Radius $R_{0}$ ) gegen Null gehen läßt.

\section{MITTEILUNGEN}

\section{Lise Meitner 70 Jahre}

$\mathrm{A}^{\mathrm{n}}$ m 7. November d. J. vollendet Prof. Lise Meitn er das 70. Lebensjahr. Viele, die ihre unveränderte Lebendigkeit und ihre Schaffenskraft auch aus den letzten Jahren kennen, werden über diese Feststellung verwundert sein. Aber die Beschäftigung mit der Physik hält offenbar jung. So war ja auch Lise
Meitners verehrter Lehrer und väterlicher Freund Max Planck noch bis hoch in seine 80-er' Jahre von einer erstaunlichen körperlichen und geist gen Frische.

Obgleich Prof. Meitner nun schon seit mehr als zehn Jahren nicht mehr in Deutschland lebt, hat sie doch den wesentlichsten Teil ihres bisherigen wissenschaft- 